

\title{
Quantitative control of idealized analysis models of thin designs
}

\author{
Ming $\operatorname{Li}^{1 \mathrm{a}}$, Junzhe Zheng ${ }^{\mathrm{a}}$, Ralph R. Martin ${ }^{\mathrm{b}}$ \\ ${ }^{a}$ State Key Laboratory of CADECG, Zhejiang University, China P.R. \\ ${ }^{b}$ School of Computer Science $\mathcal{E}$ Informatics, Cardiff University, UK
}

\begin{abstract}
When preparing a design model for engineering analysis, model idealization is often used, where defeaturing, and/or local dimension reduction of thin regions, are carried out. This simplifies the analysis, but quantitative estimates of the idealization error, the analysis error caused by this idealization, are necessary if the results are to be of practical use. The paper focuses on a posteriori estimation of such idealization error, via both a theoretical analysis and practical algorithms. Our approach can compute bounds for the errors induced by dimension reduction, defeaturing or both in combination. Performance of our error estimate is demonstrated using examples.

Keywords: defeaturing, dimension reduction, model idealization, $\mathrm{CAD} / \mathrm{CAE}$ integration, thin plate.
\end{abstract}

\section{Introduction}

Engineering analysis is typically performed using the finite element (FE) method on a mesh derived by discretizing a computer aided design (CAD)

\footnotetext{
${ }^{1}$ Corresponding author: liming@cad.zju.edu.cn 


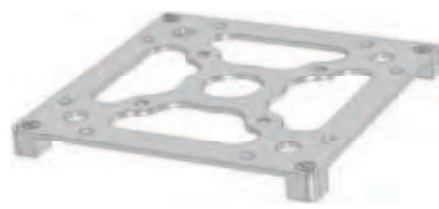

(a) Original CAD model

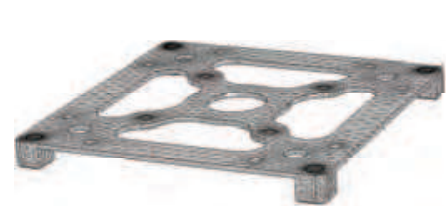

(d) Mesh for original model, 61865 elements

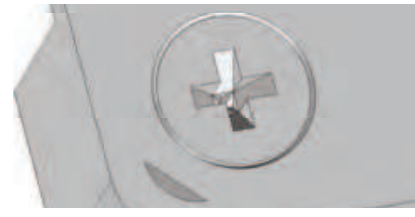

(b) Close-up of original CAD model

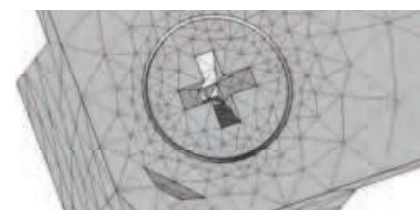

(e) Close-up of mesh for original model

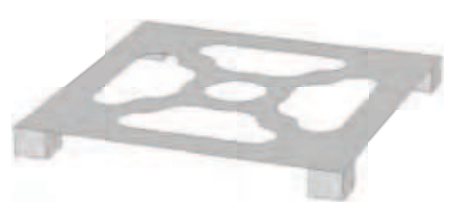

(c) Idealized CAD model

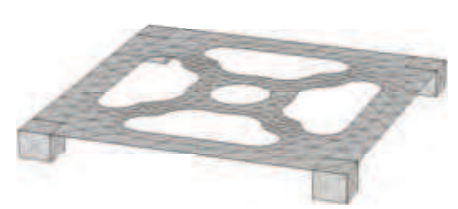

(f) Mesh for idealized model, 128 elements

Figure 1: Performing dimension reduction for the horizontal part of the original model in (a), and suppressing internal features like the one in (b), results in an idealized model (c). The latter needs far fewer mesh elements to represent it to a desired accuracy.

model. During this process, an initial step of model idealization $[1,2]$ is often performed to convert the fully detailed design to a simplified geometry, which is then used for mesh generation and FE analysis; analysis can be performed more quickly on such a model. Model idealization includes two main processes: defeaturing $[3,4]$ and dimension reduction $[5,6]$. Defeaturing removes (typically small) design features (or geometric details) that have little impact on the results of analysis, but make meshing more difficult, and cause the mesh to have a large number of elements. Dimension reduction applies classical plate or shell theories to reduce three-dimensional boundary value problems posed on a thin domain to simpler two-dimensional problems, by making assumptions about solution variation across the domain's thickness. An example of an original CAD model and a corresponding idealized model 
are shown together with corresponding analysis meshes in Figure 1.

When meshing any model, there is a need to adapt the mesh to the geometry, while FE analysis requires smooth mesh element size transitions. Regions of low thickness, and places of high curvature at small internal features, lead to a mesh for the original model with far more elements than needed for a corresponding idealized model, as shown in Figure 1. Removal of unimportant geometric details and dimension reduction of low-thickness regions significantly reduces the time and computational difficulty both for the meshing process, and the finite element analysis performed using that mesh $[3,7]$. In extreme cases, use of a fully detailed model may even lead to mesh generation failure [7] or ill-conditioned computations [8] which produce inaccurate analysis results.

The task of model idealization, however, is far from trivial. A report from Sandia National Laboratories, cited in [9], summarizes that for complex engineering designs, creation of idealized models accounts for about $60 \%$ of overall analysis time, while mesh generation accounts for about $20 \%$ and solving the boundary value problem takes about $12 \%$. Model idealization is time consuming mainly due to a lack of proper understanding of the engineering analysis errors induced by model idealization, which we will henceforth refer to as idealization errors for short. As a consequence, model idealization is usually performed manually by the mechanical or structural analysts, based on their knowledge and experience of dealing with similar analysis problems. Being able to characterize and predict idealization error are essential, both to ensure that a desired analysis accuracy can still be achieved after idealization, and as a key requirement if we are to automate the idealization 
process.

Often, model idealization produces a mixed-dimension model. Estimating idealization error has to consider possible coupling errors between regions of different dimensions, for example, a 3D region and a $2 \mathrm{D}$ plate. This important problem is extremely hard to solve in general. Here, we consider a less tricky, but still important problem: the idealization error for thin parts taken in isolation. We consider how to compute bounds on the idealization error induced by dimension reduction, defeaturing or a combination of both, as a precursor to automated methods to carry out analysis-aware thin plate model idealization.

The rest of the paper is organized as follows. Previous work is discussed in Section 2. The problem of idealization error estimation is defined in Section 3. Bounds on idealization error are derived in Section 4. Section 5 demonstrates the proposed estimator using various engineering analysis problems. Finally, conclusions are drawn in Section 6.

\section{Related work}

Various research has considered how to simplify a complex CAD model from a traditional geometry processing point of view $[1,2,6,10,11]$, but such approaches are guided solely by the geometric properties of the model (such as lengths and volumes). They do not attempt to directly estimate the effects of simplification on engineering analysis, and simply assume that geometric properties can be used as a proxy for deciding where and when it is acceptable to modify the model.

On the other hand, in the field of engineering analysis, numerous ap- 
proaches have been proposed for a posteriori estimation of FE approximation error (caused by discretization) [12] or modeling error (caused by the use of a simple mathematical model to approximate a physical phenomenon governed by a more complicated mathematical model) $[13,14,15]$. However, these techniques assume that, during analysis, the underlying geometry remains unchanged (in the sense of not being simplified, rather than just discretized), while idealization typically changes the geometry or topology of the underlying geometric model. Overall, then, the problem of idealization error estimation, in spite of its importance, is rarely studied due to its cross-disciplinary nature, and has been widely stated to be an open problem $[2,16,17,18]$.

Topological sensitivity analysis (TSA) [19, 20, 21] has been studied in design optimization, and is also closely related to the problem of estimating idealization errors, particularly defeaturing errors. These methods aim to compute the derivatives of model response quantities with respect to infinitesimal topological changes, given an existing geometry. However, design sensitivity analysis is essentially dependent on asymptotic expansions of target functionals, which strongly limits the permissible sizes and locations of the features involved. Furthermore, such approaches are not applicable to dimension reduction errors.

A few existing papers do directly address idealization error estimation, considering defeaturing $[4,22,23]$ or dimension reduction $[24,25,26,27]$ separately. The novelty of this paper is to give a unified approach for determining idealization error caused by a combination of dimension reduction and defeaturing. This does not just involve summing these two kinds of error, but 
must also consider further errors due to their interaction. Even estimating these two kinds of errors is not straightforward, as solutions are not available for the full problem (with original geometry) or for the reduced-dimension problem.

Recently, the concept of feature sensitivity analysis (FSA) was introduced for arbitrary-shaped features, for various linear problems such as the Poisson equation [22], linear elasticity [23] or plate bending [28], which estimates changes in local linear quantities when removing a single internal feature (boundary features require additional heuristics). Li et al [29] proposed a general framework for handling negative features (i.e. features in which material has been removed, such as holes) in nonlinear problems, building on previous results based on the dual weighted method (DWR) [13, 30, 31]; an adjoint error term is heuristically discarded without further consideration. The proposed estimate in this paper is derived using a strict theoretical analysis without using any heuristics for both internal and boundary negative features. Additionally, instead of approximating the errors, we determine upper bounds on them, avoiding the significant problems which may arise due to underestimating errors.

A practical approach in [4] also considers global energy errors for both negative and positive features, essentially by dividing the original problem into a simplified model and a localized model around each feature. This is similar to the idea of domain decomposition [32], but the issue of a general approach to partitioning applicable to every case remains problematic. 


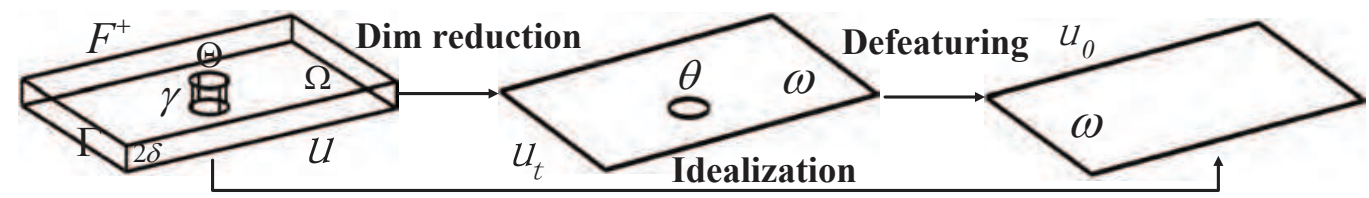

Figure 2: Original model, model after dimension reduction, and fully idealized model.

\section{Problem statement}

We now further explain and define the problem of estimation of idealization error: engineering analysis error induced by model idealization. We do so using a specific example of a thin flat CAD model containing a single negative feature (which could lie in the plate's interior or on its boundary); the problem to be solved is a Poisson equation. In general, a posteriori error estimation is very problem-dependent. We discuss in Section 4.6 how to extend the basic approach to cases with more complex geometric models, multiple features, positive features, other boundary conditions, and other physical problems.

Errors are measured in terms of changes in specific quantities of engineering interest: for example, average temperature over a local region. These are goal-oriented errors. They are estimated in an a posteriori sense by making use of the solution to the PDE problem for the idealized model, but not the solution for the original complex model.

\subsection{A specific problem}

We consider a steady-state heat conduction problem posed in a thin flat plate with an internal hole of arbitrary shape as described below; see Figure 2. Let the original plate be $\Omega-\Theta \in \mathbb{R}^{3}$, produced by creating an internal through hole $\Theta \in \Omega$ within a simple thin flat plate $\Omega$. The two regions $\Omega, \Theta$ 
are respectively $\Omega=\omega \times(-\delta, \delta)$ and $\Theta=\theta \times(-\delta, \delta)$, where $\omega, \theta \in \mathbb{R}^{2}$ are bounded domains with piecewise smooth boundaries $\partial \omega$ and $\partial \theta$, and $\delta>0$ is the half-thickness of the domain $\Omega-\Theta$.

The lateral boundaries of $\Omega$ and $\Theta$ are given in turn by $\Gamma=\partial \omega \times(-\delta, \delta)$ and $\gamma=\partial \theta \times(-\delta, \delta)$, while the top and bottom faces of $\Omega-\Theta$ are denoted by $R_{ \pm}=\left\{\left(x_{1}, x_{2}, x_{3}\right) \mid\left(x_{1}, x_{2}\right) \in \omega-\theta, x_{3}= \pm \delta\right\}$. We will use notation like $\bar{x}=\left(x_{1}, x_{2}\right)$ for $2 \mathrm{D}$ coordinates, and $x=\left(x_{1}, x_{2}, x_{3}\right)=\left(\bar{x}, x_{3}\right)$ for $3 \mathrm{D}$ coordinates.

The boundary value problem we choose to study over the plate $\Omega-\Theta$ is to find the temperature $u$ satisfying

$$
\left\{\begin{array}{lll}
L u & =0 & \text { in } \Omega-\Theta, \\
u & =0 & \text { on } \Gamma, \\
n \cdot \nabla u=f^{ \pm} & \text {on } R^{ \pm} \\
n \cdot \nabla u=0 & \text { on } \gamma,
\end{array}\right.
$$

where

$$
L u=-\nabla \cdot(\nabla u)
$$

$f^{ \pm}$are the prescribed heat fluxes (independent of $u$ ) across the top and bottom faces $R^{ \pm}$, and $n$ is an outward normal vector to the corresponding model boundary.

The space of admissible functions for the problem in Eq. (1) is

$$
V=\left\{v \in H^{1}(\omega-\theta) \times H^{1}((-1,1)):\left.v\right|_{\partial \omega}=0\right\},
$$

where $H^{1}(\omega-\theta), H^{1}((-1,1))$ are the standard Sobolev spaces.

The idealization error may be estimated by using a weak form of Eq. (1), which may be derived by multiplying both sides of the first equation by a 
function $v \in V$ and then performing integration by parts, taking boundary conditions into account. The corresponding equivalent weak form of Eq. (1) seeks to find the solution $u \in V$ such that

$$
\alpha(u, v)=l(v), \quad \text { for } v \in V
$$

where

$$
\alpha(u, v)=\int_{\omega-\theta} \int_{-1}^{1}\left\{\frac{1}{\delta} \frac{\partial u}{\partial z} \frac{\partial v}{\partial z}+\delta \nabla_{\bar{x}} u \cdot \nabla_{\bar{x}} v\right\} d z d \bar{x}
$$

and

$$
l(v)=\int_{\omega-\theta}\left(f^{+}(\bar{x}) v(\bar{x}, 1)+f^{-}(\bar{x}) v(\bar{x},-1)\right) d \bar{x}
$$

the notation $\nabla_{\bar{x}}$ means $\left(\partial / \partial x_{1}, \partial / \partial x_{2}\right)$.

\subsection{The idealized problem}

The idealized problem comes from a combination of dimension reduction and defeaturing. Firstly, the dimension reduction process assumes that the solution does not vary across the thickness, i.e. $\partial u / \partial z=0$. Secondly, the defeaturing process suppresses feature $\Theta$ from model $\Omega-\Theta$. As a result, the original problem in Eq. (2) is converted to a new analysis problem: find the solution $u_{0}$ satisfying

$$
\left\{\begin{array}{lll}
L_{0} u_{0} & =f_{0} & \text { in } \omega \\
u_{0} & =0 & \text { on } \partial \omega
\end{array}\right.
$$

where

$$
L_{0} u_{0}=-2 \delta \nabla_{\bar{x}} \cdot\left(\nabla_{\bar{x}} u_{0}\right)
$$

As feature $\Theta$ has been removed, the boundary conditions prescribed over $\Theta$ in Eq. (1) also disappear. This new analysis problem in Eq. (3) is now defined over the 2D mid-surface $\omega$. 
Equivalently, we may write: find the solution $u_{0} \in V_{0}$ such that

$$
\alpha_{0}\left(u_{0}, v\right)=l_{0}(v), \text { for } v \in V_{0},
$$

where

$$
\begin{array}{r}
\alpha_{0}\left(u_{0}, v\right)=2 \delta \int_{\omega} \nabla_{\bar{x}} u_{0} \cdot \nabla_{\bar{x}} v d \bar{x}, \\
=-2 \delta \int_{\omega} \nabla_{\bar{x}} \cdot\left(\nabla_{\bar{x}} u_{0}\right) v d \bar{x}, \\
l_{0}(v)=\int_{\omega} f_{0} v(\bar{x}) d \bar{x}, \quad \text { with } f_{0}=f^{+}+f^{-},
\end{array}
$$

and

$$
V_{0}=\left\{v \in H^{1}(\omega) \times H^{1}((-1,1)): \frac{\partial v}{\partial z}=0,\left.v\right|_{\partial \omega}=0\right\} .
$$

Note that the solution $u_{0}$ is defined via a $2 \mathrm{D}$ problem over the mid-surface $\omega$. Its extension to its $3 \mathrm{D}$ counterpart over $\Omega$ is trivial due to the assumption that the 3D solution is constant in the thickness direction. We still denote this $3 \mathrm{D}$ solution by $u_{0}$ without confusion, allowing us to also say that this solution is defined over the $3 \mathrm{D}$ model $\Omega$ whenever needed.

\subsection{An intermediate problem}

To facilitate derivation of our estimate of the idealization error in the next Section, an intermediate problem is now introduced and defined. Model idealization is considered to be a two-step process which first performs dimension reduction, resulting in an intermediate model, and then defeatures the dimensionally reduced model, resulting in the final idealized model; again see Figure 2. Note that we do not actually need to compute a solution to this intermediate problem to estimate the final idealization error. 
The solution to the intermediate analysis problem is denoted $u_{t}$. Performing dimension reduction, and letting $\partial u / \partial z=0$ in Eq. (1), gives the problem: find the solution $u_{t}$ such that

$$
\left\{\begin{array}{lll}
L_{0} u_{t} & =f_{0} & \text { in } \omega-\theta, \\
u_{t} & =0 & \text { on } \partial \omega, \\
n \cdot \nabla_{\bar{x}} u_{t}=0 & \text { on } \partial \theta .
\end{array}\right.
$$

Note that the interior operator $L_{0}$ is the same as in Eq. (3), while a new boundary condition over $\partial \theta$ is prescribed.

Equivalently, we may write: find solution $u_{t} \in V_{t}$ such that

$$
\alpha_{t}\left(u_{t}, v\right)=l_{t}(v), \text { for } v \in V_{t},
$$

where

$$
\begin{gathered}
\alpha_{t}\left(u_{t}, v\right)=2 \delta \int_{\omega-\theta} \nabla_{\bar{x}} u_{t} \cdot \nabla_{\bar{x}} v d \bar{x} \\
l_{t}(v)=\int_{\omega-\theta} f_{0} v(\bar{x}) d \bar{x}, \quad V_{t}=\left\{v \in V, \frac{\partial v}{\partial z}=0\right\} .
\end{gathered}
$$

Again, $u_{t}$ is computed over the 2D mid-surface, now $\omega-\theta$, and may also be extended to $\Omega-\Theta$ in $3 \mathrm{D}$.

\subsection{Idealization error for quantities of interest}

The process of idealization changes the mathematical model from operator $L$ to operator $L_{0}$ due to dimension reduction, while defeaturing changes its computational domain, from $\Omega-\Theta$ to $\Omega$, or $\omega$. These differences cause the corresponding solutions $u$ and $u_{0}$ to differ, affecting the accuracy of the engineering analysis. 
This difference affects local quantities of interest as we now explain. Engineering analysis error, caused by FE approximation or different mathematical models alike, was originally measured globally in terms of differences in quantities such as global energies. Global measures are, however, insensitive to local quantities of interest such as local temperatures, fluxes, etc. Goaloriented error measures provide a better way of characterizing error [33], and are used to study idealization error here.

Following [24, 25], local quantities of interest can generally be expressed as continuous linear functionals $Q(\cdot)$ of the following form, on the space of admissible functions $V$ :

$$
Q(v)=\int_{\omega}\left(g^{+}(\bar{x}) v(\bar{x}, 1)+g^{-}(\bar{x}) v(\bar{x},-1)\right) d \bar{x}
$$

with $g^{ \pm} \in L^{2}(\omega)$, i.e. the Lebesgue space of square-integrable functions over $\omega$. In other words, we are interested in the accuracy of the solution $u_{0}$ of the idealized model on those portions of the top and bottom surfaces where $g^{ \pm}$ does not vanish.

As solutions $u$ and $u_{0}$ differ, this in turn affects any quantity of interest, $Q\left(u_{0}\right)$ and $Q(u)$. Their difference is the idealization error:

$$
e=Q(u)-Q\left(u_{0}\right)
$$

We estimate this error a posteriori by using solution $u_{0}$ without explicitly finding $u$. Practical computation of solution $u_{0}$ will involve certain numerical approximation errors. The relation between the idealization error under study to the approximation error will be discussed in Section 6 .

We now have a clear mathematical definition of idealization error in Eq. (8) in terms of the original problem in Eq. (2) and its idealized ver- 
sion in Eq. (4). Additionally, an intermediate problem has been defined in Eq. (6), allowing the next section to detail our concrete approach for finding a bound on the idealization error.

\section{Computable bounds for idealization errors}

We now give our unified approach for estimating goal-oriented idealization error (which may include defeaturing errors and/or dimension reduction errors). The key idea is to first convert the problem to one of estimating a unified modeling error defined over the same geometry, which can then be re-expressed in a residual form using adjoint solutions [12] as weight factors. We then partition the re-formulated idealization error into three different terms: a computable term based on the solution defined over the idealized model, a dimension reduction residual and a defeaturing residual. By finding bounds on these two residual terms, a computable bound can be derived for the idealization error, as we now explain.

\subsection{From idealization error to modeling error}

As the original model $\Omega-\Theta$ is contained within the idealized model $\Omega$, i.e. $\Omega-\Theta \subset \Omega$, the solution $u_{0}$ is well defined over the geometry $\Omega-\Theta$. It is then not difficult to see that the solution $u_{0}$ is equal to the following solution $\hat{u}_{0}$ over the region $\Omega-\Theta$ (defined over its mid-surface $\left.\omega-\theta\right)$ with certain Neumann conditions prescribed over the internal boundary $\partial \theta$ :

$$
\left\{\begin{array}{lll}
L_{0} \hat{u}_{0} & =f_{0} & \text { in } \omega-\theta \\
\hat{u}_{0} & =0 & \text { on } \partial \omega \\
n \cdot \nabla \hat{u}_{0} & =h_{0} & \text { on } \partial \theta
\end{array}\right.
$$


for

$$
h_{0}=\frac{\partial u_{0}}{\partial n}
$$

The corresponding weak formulation for Eq. (9) is: find the solution $\hat{u}_{0} \in V_{t}$ such that

$$
\hat{\alpha}_{0}\left(\hat{u}_{0}, v\right)=l_{t}(v), \quad \text { for } v \in V_{t}
$$

with

$$
\hat{\alpha}_{0}\left(\hat{u}_{0}, v\right)=2 \delta\left(\int_{\omega-\theta} \nabla_{\bar{x}} \hat{u}_{0} \cdot \nabla_{\bar{x}} v d \bar{x}-\int_{\partial \theta} h_{0} v d s\right) .
$$

Equality of $u_{0}$ and $\hat{u}_{0}$ over $\omega-\theta$ shows that

$$
e=Q(u)-Q\left(u_{0}\right)=Q(u)-Q\left(\hat{u}_{0}\right)
$$

By this process, the idealization error defined over different geometries $\Omega-\Theta$ and $\omega$ (on the left-hand side) has been converted into a modeling error defined over the same geometry $\Omega-\Theta$ (on the right-hand side).

\subsection{Modeling error estimation}

The modeling error $Q(u)-Q\left(\hat{u}_{0}\right)$ can be estimated using adjoint theory. For a specific primal analysis problem, for example, the one in Eqs. (2) and (10), and a target functional $Q(\cdot)$, an adjoint problem can be defined [12]. Its corresponding adjoint solutions are typically used as weight factors in $a$ posteriori goal-oriented error estimation techniques. For a good introduction to adjoint theory, see [34].

The adjoint problems for the primal problems in Eqs. (2) and (10) with respect to a linear target function $Q(\cdot)$ are: find solutions $p \in V, \hat{p}_{0} \in V_{t}$ such that

$$
\alpha(q, v)=Q(v), \quad v \in V
$$


and

$$
\hat{\alpha}_{0}\left(v, \hat{p}_{0}\right)=Q(v), \quad v \in V_{t} .
$$

Define the residual $R(u, v)=l(v)-\alpha(u, v)$. From the equality $\hat{u}_{0}=u_{0}$ over $\Omega-\Theta$, we then have

$$
\begin{aligned}
Q(u)-Q\left(\hat{u}_{0}\right) & =a(u, p)-a\left(\hat{u}_{0}, p\right) \\
& =l(p)-a\left(\hat{u}_{0}, p\right) \\
& =R\left(u_{0}, p\right) .
\end{aligned}
$$

The result is summarized below.

Lemma 1. The idealization error defined by Eq. (8) can be expressed as below, assuming a linear quantity of interest $Q(u)$ defined by Eq. (7):

$$
Q(u)-Q\left(u_{0}\right)=R\left(u_{0}, p\right)
$$

where $p$ is the adjoint solution defined via Eq. (11).

\subsection{Residual terms}

The residual $R\left(u_{0}, p\right)$ in Lemma 1 can be partitioned into three different terms, which we handle separately.

Writing

$$
R(u, v)=\alpha(u, v)-l(v), \quad R_{t}(u, v)=\alpha_{t}(u, v)-l_{t}(v)
$$

it can be easily verified that

$$
Q(u)-Q\left(u_{0}\right)=R\left(u_{0}, p\right)=R\left(u_{0}, p_{0}\right)+R\left(u_{0}, p-p_{0}\right)
$$


$Q(u)-Q\left(u_{0}\right)=R\left(u_{0}, p_{0}\right)+R\left(u_{0}, p_{t}-p_{0}\right)+R\left(u_{t}, p-p_{t}\right)+R\left(u_{0}-u_{t}, p-p_{t}\right)$.

As $p_{t}=p_{0}$ for $z= \pm 1$, and $\partial u_{0} / \partial z=0$, it follows that $R\left(u_{0}, v\right)=R_{t}\left(u_{0}, v\right)$. Similarly, $R\left(u_{0}, p_{0}\right)=R_{t}\left(u_{0}, p_{0}\right)$. Additionally, $R\left(u_{0}-u_{t}, p-p_{t}\right)=R_{t}\left(u_{0}-\right.$ $\left.u_{t}, p-p_{t}\right)=0$, leading to the following lemma:

Lemma 2. The idealization error can be expressed as a sum of three terms:

$$
Q(u)-Q\left(u_{0}\right)=R_{t}\left(u_{0}, p_{0}\right)+R_{t}\left(u_{0}, p_{t}-p_{0}\right)+R\left(u_{t}, p-p_{t}\right)
$$

where $R_{t}\left(u_{0}, p_{0}\right)$ is readily computable from the solution to the idealized problem, $R\left(u_{t}, p-p_{t}\right)$ is the dimension reduction residual, and $R_{t}\left(u_{0}, p_{t}-p_{0}\right)$ is the defeaturing residual.

In the above equation, the first term $R_{t}\left(u_{0}, p_{0}\right)$ can be easily evaluated using the primal and adjoint solutions $u_{0}, p_{0}$ defined over model $\omega$. Thus, to estimate $Q(u)-Q\left(u_{0}\right)$, we need to simply estimate the dimension reduction residual $R\left(u_{t}, p-p_{t}\right)$ and the defeaturing residual $R_{t}\left(u_{0}, p_{t}-p_{0}\right)$, which we consider next.

\subsection{Bounds for the defeaturing residual}

Estimating the defeaturing residual $R_{t}\left(u_{0}, p_{t}-p_{0}\right)$ is considered in this section. The following result shows how to convert the residual into a boundary integral:

Lemma 3. The defeaturing residual may be rewritten as

$$
R_{t}\left(u_{0}, p_{t}-p_{0}\right)=2 \delta \int_{\partial \theta} h_{0}\left(p_{t}-p_{0}\right) d s, \quad \text { where } h_{0}=\frac{\partial u_{0}}{\partial n} .
$$


Proof: From the definitions of $R_{t}(\cdot, \cdot)$ and Eqs. (10) and (6), we have

$$
\begin{aligned}
R_{t}\left(u_{0}, v\right) & =\alpha_{t}\left(u_{0}, v\right)-l_{t}(v) \\
& =\alpha_{t}\left(u_{0}, v\right)-\hat{\alpha}_{t}\left(u_{0}, v\right) \\
& =2 \delta \int_{\partial \theta} h_{0} v d s .
\end{aligned}
$$

Replacing $v$ by $p_{t}-p_{0}$ completes the proof.

The above Lemma shows that the defeaturing residual completely depends on the adjoint error $p_{t}-p_{0}$ over the internal boundary $\partial \theta$. It is not difficult to verify for $p_{t}-p_{0}$ that

$$
\int_{\omega-\theta} \nabla\left(p_{t}-p_{0}\right) \cdot \nabla v d V=\int_{\partial \theta} d_{0} v d s, \text { for, } d_{0}=\frac{\partial p_{0}}{\partial n} .
$$

Thus, the following bound can be deduced for the defeaturing residual.

Theorem 1. The defeaturing residual is bounded as follows:

$$
R_{t}\left(u_{0}, p_{t}-p_{0}\right) \leq 2 \delta C^{2}\left\|h_{0}\right\|_{L^{2}(\partial \theta)}\left\|d_{0}\right\|_{L^{2}(\partial \theta)},
$$

where $C$ is a constant only dependent on the geometry of region $\Omega-\Theta$, and

$$
h_{0}=\frac{\partial u_{0}}{\partial n}, \quad d_{0}=\frac{\partial p_{0}}{\partial n} .
$$

We discuss the value of $C$ later.

Proof: Define

$$
\|v\|_{E(\omega-\theta)}=\int_{\omega-\theta} \nabla v \cdot \nabla v d s=\frac{\alpha_{t}(v, v)}{2 \delta} .
$$

From the Trace Theorem, a const $C$ exists such that for $v \in V$,

$$
\|v\|_{L^{2}(\partial(\omega-\theta))} \leq C\|v\|_{E(\omega-\theta)},
$$


where $C$ is a positive constant only dependent on the geometry of region $\Omega-\Theta$. Then,

$$
\begin{aligned}
\left|\alpha_{t}\left(p_{t}-p_{0}, v\right)\right| & =2 \delta\left|\int_{\partial \theta} d_{0} v d s\right| \\
& \leq 2 \delta\left\|d_{0}\right\|_{L^{2}(\partial \theta)}\|v\|_{L^{2}(\partial \theta)} \\
& \leq 2 \delta\left\|d_{0}\right\|_{L^{2}(\partial \theta)}\|v\|_{L^{2}(\partial(\omega-\theta))} \\
& \leq 2 \delta\left\|d_{0}\right\|_{L^{2}(\partial \theta)} C\|v\|_{E(\omega-\theta)} .
\end{aligned}
$$

Replacing $v=p_{t}-p_{0}$ in the above equation, we thus obtain

$$
\begin{aligned}
2 \delta\left\|p_{t}-p_{0}\right\|_{E(\omega-\theta)}^{2} & =\alpha_{t}\left(p_{t}-p_{0}, p_{t}-p_{0}\right) \\
& \leq 2 \delta\left\|d_{0}\right\|_{L^{2}(\partial \theta)} C\left\|p_{t}-p_{0}\right\|_{E(\omega-\theta)}
\end{aligned}
$$

or equivalently

$$
\left\|p_{t}-p_{0}\right\|_{E(\omega-\theta)} \leq C\left\|d_{0}\right\|_{L^{2}(\partial \theta)}
$$

Thus from Lemma 3,

$$
\begin{aligned}
\left|R_{t}\left(u_{0}, p_{t}-p_{0}\right)\right| & \leq 2 \delta\left\|h_{0}\right\|_{L^{2}(\partial \theta)}\left\|p_{t}-p_{0}\right\|_{L^{2}(\partial \theta)} \\
& \leq 2 \delta C\left\|h_{0}\right\|_{L^{2}(\partial \theta)}\left\|p_{t}-p_{0}\right\|_{E(\omega-\theta)} \\
& \leq 2 \delta C^{2}\left\|h_{0}\right\|_{L^{2}(\partial \theta)}\left\|d_{0}\right\|_{L^{2}(\partial \theta)},
\end{aligned}
$$

completing the proof.

The above derivation shows that the constant $C$ should ideally be set to

$$
C=\frac{\left\|p_{t}-p_{0}\right\|_{L^{2}(\partial \theta)}}{\left\|p-p_{0}\right\|_{E(\omega-\theta)}}
$$

However, since the solution $p$ is not available, instead we may in practice use an estimate of

$$
C=\lambda \frac{\left\|p_{0}\right\|_{L^{2}(\partial(\omega-\theta))}}{\left\|p_{0}\right\|_{E(\omega-\theta)}}
$$


where $\lambda$ is a balancing constant set to the ratio of the measure (length or area) of the overall non-homogeneous Dirichlet boundary to that of the feature boundary $\partial \theta$. The reason behind this can be observed from the different numerators of the above equations, respectively defined over $\partial \theta$ and $\partial(\omega-\theta)$. $\lambda$ aims to balance such area differences.

\subsection{Bounds for the dimension reduction residual}

Estimation of the dimension reduction residual $R\left(u_{t}, p-p_{t}\right)$ is discussed next. Unlike previous approaches to dimension reduction error, in this specific problem, none of the solutions $u_{t}, p_{t}$, or $p$ are known. We must estimate dimension reduction error a priori without use of any pre-computed solutions. We do so using previous results from [24, 25].

Let

$$
f^{o}=\left(f^{+}-f^{-}\right) / 2, \quad f^{e}=\left(f^{+}+f^{-}\right) / 2 .
$$

Lemma 4. The dimension reduction error $e_{0}=u-u_{t}$ is bounded globally as follows:

$$
\left\|e_{0}\right\|_{E(\Omega-\Theta)}^{2} \leq \zeta_{u p p}^{2}=\frac{2 \delta}{3}\left\|f^{e}\right\|_{L^{2}(\Omega-\Theta)}^{2}+2 \delta\left\|f^{o}\right\|_{L^{2}(\Omega-\Theta)}^{2} .
$$

Proof: The proof can be obtained using the Peano Kernel Theorem [35], by following similar lines to those used in [24].

Similarly,

$$
\left\|p-p_{0}\right\|_{E(\Omega-\Theta)}^{2} \leq \bar{\zeta}_{u p p}^{2}=\frac{2 \delta}{3}\left\|g^{e}\right\|_{L^{2}(\Omega-\Theta)}^{2}+2 \delta\left\|g^{o}\right\|_{L^{2}(\Omega-\Theta)}^{2}
$$

for

$$
g^{o}=\left(g^{+}-g^{-}\right) / 2, g^{e}=\left(g^{+}+g^{-}\right) / 2,
$$


where $g^{+}, g^{-}$are defined in Eq. (7).

Using these we arrive at the following bounds on the dimension reduction residual:

Theorem 2. The dimension reduction residual $R\left(u_{t}, p-p_{t}\right)$ is bounded as follows:

$$
R\left(u_{t}, p-p_{t}\right) \leq \zeta_{u p p} \bar{\zeta}_{u p p}
$$

Proof:

$$
\begin{aligned}
\left|R\left(u_{t}, p-p_{t}\right)\right| & =\left|l\left(p-p_{t}\right)-\alpha\left(u_{t}, p-p_{t}\right)\right| \\
& =\left|\alpha\left(u, p-p_{t}\right)-\alpha\left(u_{t}, p-p_{t}\right)\right| \\
& =\left|\alpha\left(u-u_{t}, p-p_{t}\right)\right| \\
& \leq\left\|u-u_{t}\right\|_{E(\Omega-\Theta)}\left\|p-p_{t}\right\|_{E(\Omega-\Theta)} \\
& \leq \zeta_{u p p} \bar{\zeta}_{u p p}
\end{aligned}
$$

Combining Lemma 2, and Theorems 1 and 2 gives the following overall theorem bounding idealization error:

Theorem 3. The idealization error defined by Eq. (8) is bounded below by

$$
Q(u)-Q\left(u_{0}\right) \leq R_{t}\left(u_{0}, p_{0}\right)+\zeta_{u p p} \bar{\zeta}_{u p p}+C^{2} 2 \delta\left\|h_{0}\right\|_{L^{2}(\partial \theta)}\left\|d_{0}\right\|_{L^{2}(\partial \theta)}
$$

\subsection{Discussion}

We now discuss potential limitations of the proposed bounds, and its possible extension to other general cases. Our approach depends on the following four main steps to achieve the error bounds: (I) converting idealization error into modeling error based on Eq. (9) in Section 4.1, (II) re-formulating modeling error based on adjoint theory (Theorem 1) in Section 4.2, (III) building 
bounds on the dimension reduction residual based on Theorem 2, and (IV) building bounds on the defeaturing error residual based on Theorem 1 .

Specifically, step (I) works as long as the original model is contained within the idealized/simplified model. This means all features must be negative (i.e. remove material). Step (II) works as long as the primal and adjoint solutions over the original model and over the idealized model lie in the same solution space. Removing features with Dirichlet boundary conditions (or solution values are set along boundary) will result in different solution spaces, in which case our method cannot be used. The first two steps work for both defeaturing and dimension reduction.

Step (III) applies to dimension reduction. Basically, the dimension reduction process involves a process of building the analysis problem over an extracted mid-surface (from a thin model of constant or varying thickness). If boundary conditions over the lateral faces are not constant across the thickness, it is not a trivial issue to set corresponding boundary conditions for the mid-surface boundary, which is why we assume free lateral boundary conditions for the original problem in Eq. (1). For the same reason, the local quantity takes the form in Eq. (7) so that it can also be easily defined over the idealized model $\omega$. If this issue can be resolved, specific geometric forms (for example, plates of varying thickness) are not essential as long as the analysis problem for the idealized model is well defined. By utilizing other approaches to estimating the dimension reduction error for other kinds of shells [26], our proposed technique can be extended to further cases.

Step (IV) also works to bound the error even when simultaneously removing multiple negative features. This is because it does not need additional 
computations provided the primal and adjoint solutions $u_{0}, p_{0}$ are computed from the idealized model; furthermore, the constant $C$ does not depend on concrete solutions. Thus, in the case of multiple features, the residual estimate is found simply by summing the derived error bounds together.

In summary, the accuracy of the derived error bound in Theorem 3 depends on the three different terms on the right hand side of Eq. (16). The first term $R_{t}\left(u_{0}, p_{0}\right)$ can be exactly evaluated (without estimation) once the solutions $u_{0}, p_{0}$ have been computed, ignoring any numerical approximation errors. This term generally accounts for the largest contribution to the overall estimated idealization error, and thus a final idealization error estimation with high accuracy can be generally expected. The other two residual terms are estimates and may reduce the accuracy of the final idealization error estimate. Specifically, accuracy of the dimension reduction residual, i.e. the second term on the right hand side of Eq. (16), depends on those of the energy norms of the primal and adjoint solutions; See also Eq. (15). Nice experimental performance has been observed for such error estimates in [25], which may generally further ensure the accuracy of the second estimated term. In rare cases, $u-u_{t}$ and $p-p_{t}$ may have different signs, and the term may significantly overestimate the error. It can be further refined by using the parallelogram identity $[25,36]$. The last term involves no approximation, but does depend on a constant $C$ whose value must be determined. Essentially, no theoretically sound approach exists for finding such a constant except in a very few special cases. In Section 4.4 we provide a simple heuristic approach for setting this value. We note that obtaining an exact or tight value for constant $C$ is not essential due to the dominant contribution of the first term 
to the overall idealization error. Our experiments in the next section verify this assertion. Nevertheless, a reliable strategy for giving a value to $C$ based on solutions $u_{0}, p_{0}$ needs further research.

\section{Experimental results}

Applying Theorem 3 for idealization error estimation is straightforward to implement for a single feature. We compute the primal and adjoint solutions $u_{0}, p_{0}$ over the idealized model $\omega$ with a sufficiently dense mesh (ignoring numerical approximation errors), and then evaluate the error bounds based on the theorem. Our approach for estimation of idealization error has been implemented on a $2.8 \mathrm{GHz}$ dual quad-core processor with $4 \mathrm{~GB}$ RAM using COMSOL [37], a commercial finite element based CAD/CAE system. We have tested the approach's accuracy for 2D defeaturing cases in Section 5.1, for 3D idealization in Section 5.2, and finally for a complex example with multiple features in Section 5.3.

The accuracy of an error estimate is usually measured in terms of effectivity index, defined as the ratio between the estimated error $e$ and the exact error $E$, that is,

$$
I=e / E .
$$

For goal-oriented error estimation as considered in this paper, it is generally believed to be difficult or expensive to obtain highly accurate error estimates [38]. In practice, effectivity index values up to 10 can still be useful [39]. Nevertheless, Estep has provided accurate and inexpensive computable goal-oriented error estimates [40]. We leave the reader to form their own judgement on the results reported here. 
In these examples, the exact error $E=Q(u)-Q\left(u_{0}\right)$, denoted Exact, is evaluated in practical terms by directly computing the field solutions $u, u_{0}$ from Eqs. (1) and (3) over sufficiently dense meshes. We ignore any discrete FE approximation errors, and use the resulting values as ground truth for comparisons.

\subsection{Defeaturing error estimates}

Idealization error is a combination of dimension reduction error, and defeaturing error. Performance of our dimension reduction error has been demonstrated in previous work $[24,25]$. We thus first show here the performance of the defeaturing bounds we have derived (denoted $U p p$ ) based on Theorem 1, for an analysis problem defined over a 2D example in Figure 3, in the same form as Eq. (5). The const $C$ involved in evaluating $U p p$ was computed via Eq. (14). We compare the derived Upp to the ground truth, denoted Exact as explained above, and the error $R_{t}\left(u_{0}, p_{0}\right)$ without further approximations, denoted $A p p$.

The model in Figure 3 contains an internal elliptic hole $F_{1}$ with semimajor and semiminor axes of $a$ and $a / 2$, and a boundary circular feature $F_{2}$ of radius $b$; values of $a, b$ are explained later. In this example, the internal boundary was set free, and other outer boundary conditions are depicted in Figure 3. The quantity of interest is defined over a circular region $S$ with radius 0.2 to be

$$
Q(u)=\int_{S} u d S .
$$

In our tests, we took feature $F_{1}$ or $F_{2}$ as the target negative feature to be suppressed. First consider feature $F_{1}$. Figure $4(\mathrm{a})$ shows for the quantity of 


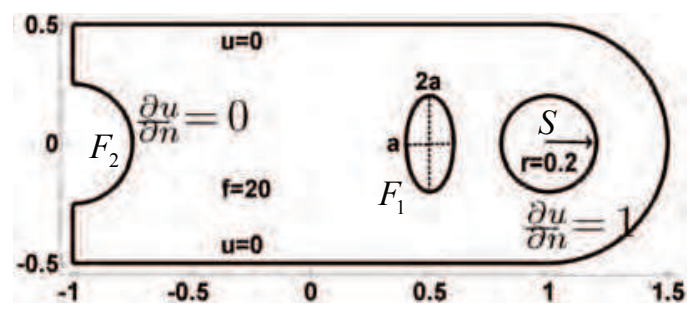

Figure 3: A defeaturing error estimation problem.

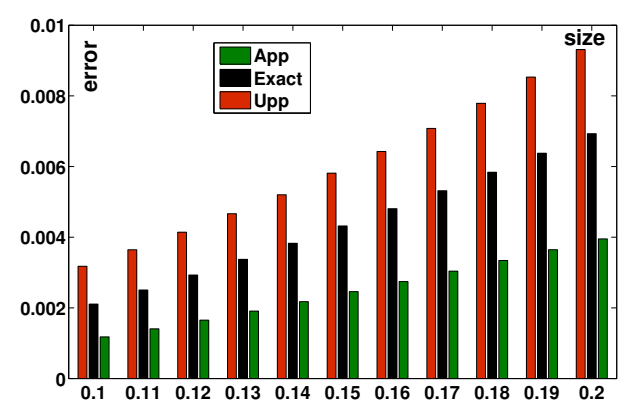

(a) Error comparisons

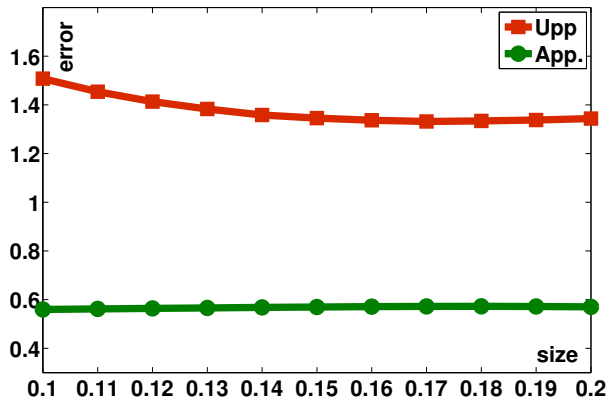

(b) Effectivity index

Figure 4: Idealization error estimates for the internal elliptic hole shown in Figure 3.

interest how the estimated error bound Upp and the estimated error App vary with the feature's semimajor axis a, compared to the ground truth Exact; values of $a$ were varied from 0.1 to 0.2 with a step size of 0.01 . Results are compared using effectivity indices plotted in Figure 4(b). As can be seen, our estimates provide close upper bounds for the exact value with effectivity indices ranging between 1.3 and 1.6, demonstrating that it is highly effective in estimating defeaturing error.

Next consider feature $F_{2}$. Similar experimental results for changes in the boundary circular feature are summarized in Figure 5 ( $\lambda$ in Eq. (14) is set 1 in this example); values of $b$ were varied from 0.1 to 0.3 with a step size of 0.02 in this test. Again, our error estimates bound the exact value and are 


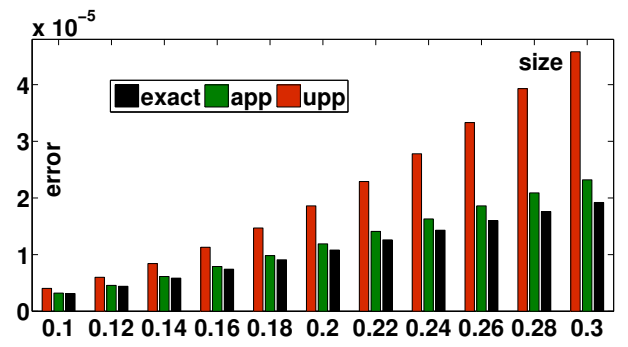

(a) Error comparisons

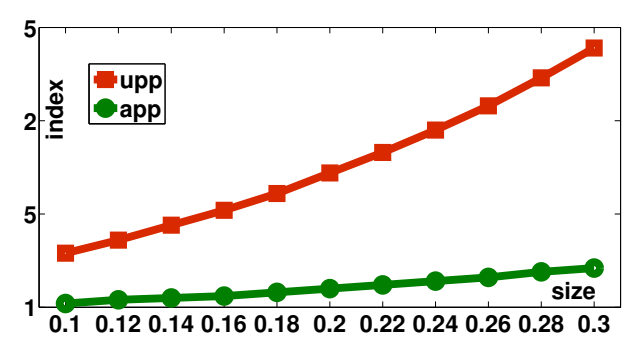

(b) Effectivity index

Figure 5: Idealization error estimates for the boundary circular hole shown in Figure 3.

close to it, with effectivity indices ranging from 1.3 to 2.5 as the radius of the circular feature changes.

\subsection{Idealization error estimates}

Our approach was also tested on the example in Figure 6 to determine its effectivity in estimating idealization error caused by a combination of dimension reduction and defeaturing. The internal hole is taken as the target feature to be suppressed. The example follows the definition in Section 3, and is not further explained here. The quantity of interest is defined over the top face $R^{+}$as

$$
Q(u)=\int_{R^{+}} u d S .
$$

In this example, effectivity of the idealization error estimate was tested under simultaneously changes of both the plate's height and the internal hole's size, thus forming an error surface shown in Figure 7(a). In this example, besides the estimated upper bounds Upp and the ground truth values Exact, we also computed for comparison the approximated error as $R_{t}\left(u_{0}, p_{0}\right)$ and lower bounds set as $R_{t}\left(u_{0}, p_{0}\right)-\zeta_{u p p} \bar{\zeta}_{u p p}-C^{2}\left\|h_{0}\right\|_{L^{2}(\partial \theta)}\left\|d_{0}\right\|_{L^{2}(\partial \theta)}$ and denoted Low. 


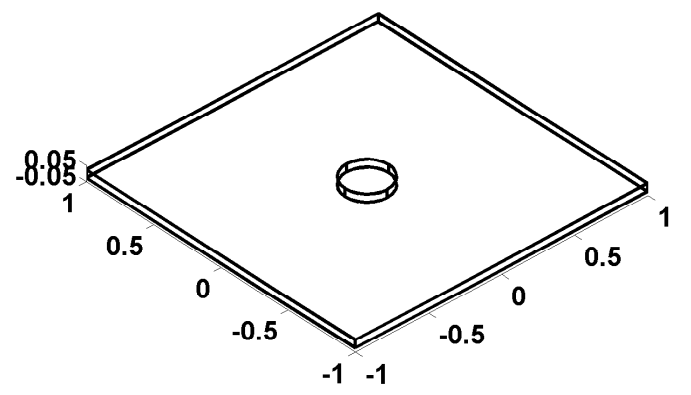

Figure 6: Example of an idealization error estimation problem.

The estimated values, including its lower and upper bounds, are compared to the ground truth in Figure 7(a), and the corresponding effectivity indices are plotted in Figure 7(b). As can be seen from Figure 7(a), the estimated upper and lower errors bound the ground truth errors, with corresponding effectivity indices ranging from 1.0 to 2.7 , and 0.3 to 1.0 . Furthermore, the estimated value also approximates the ground truth with effectivity indices ranging from 0.4 to 1.0. All demonstrate the effectivity of our error estimation approach. Strangely, the accuracy of error estimates was worse for small values of the heights and the features' sizes. This was perhaps due to discretization errors that are assumed ignorable, but it is hard to verify this claim due to the computational requirements. When the errors are small, discretization error plays a major role, and it is harder to estimate the idealization error accurately. This situation can be worse when a locking phenomenon happens in solving a very thin structure [41]. Results could be improved by further considering the numerical approximation error involved in computing $u_{0}$, as further discussed in Section 6 . 


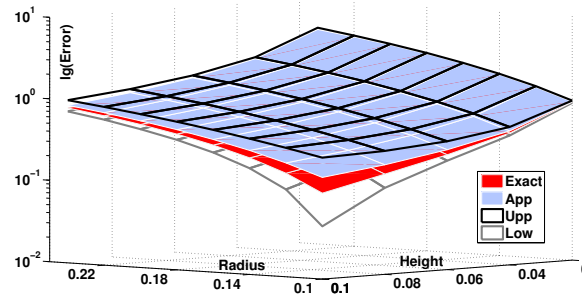

(a) Error comparisons

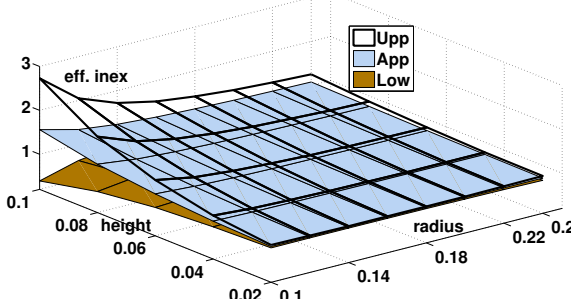

(b) Effectivity index

Figure 7: Idealization error estimation results for the problem in Figure 6.

\subsection{Multiple features}

We also tested the accuracy of our approach for the complex plate model in Figure 8, which represents a simplified version of the model in Figure 1(a). The model's symmetry allows us to concentrate on four features labeled $F_{1}, \ldots, F_{4}$; other features of larger size are not studied here. Meshes of the original thin model are shown in Figure 8(b). The analysis problem is defined following Section 5.2.

In this test, we took the idealized model as the mid-surface by removing the 20 features including $F_{1}, \ldots, F_{4}$ and their symmetric counterparts, as shown in Figure 9. An error bound was computed for each feature based on Theorem 3. The estimated bounds were compared with the ground truth values in Figure 10. As can be seen these computed values bound the ground truth value tightly with effectivity indices ranging from 1.0 to 1.2. Based on the these computational results, all 20 features can be removed, leaving an idealized 2D model in Figure 9. 


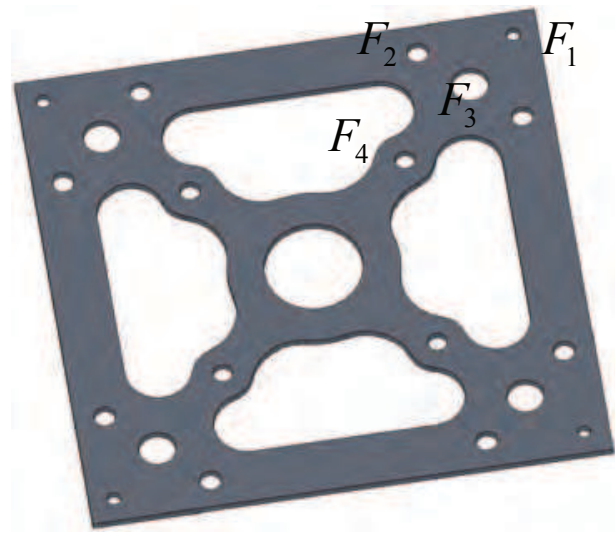

(a) Original plate

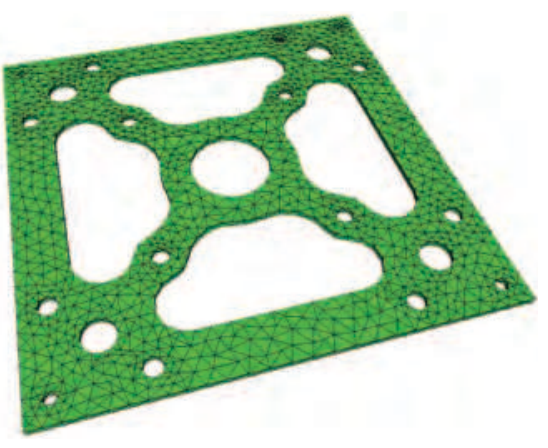

(b) Original mesh model

Figure 8: Example of an idealization error estimation problem for cases of multiple features.

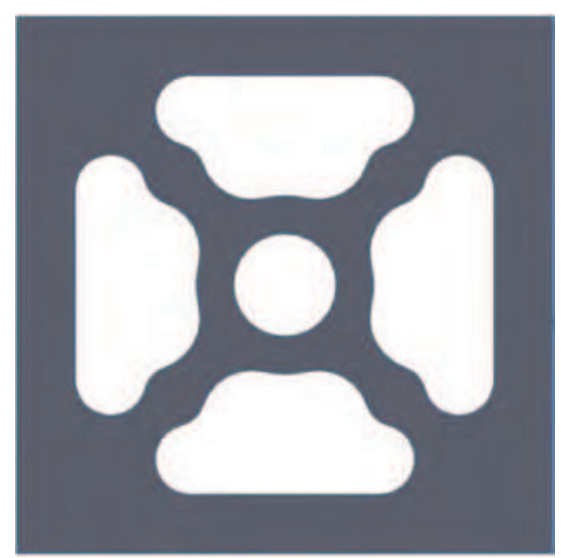

(a) Idealized plate

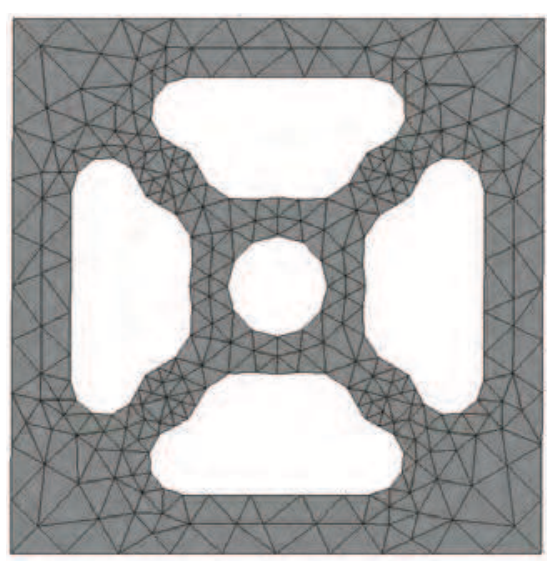

(b) Mesh model of idealized plate

Figure 9: Idealized model and its mesh form for the model in Figure 8.

\section{Conclusion and future work}

The paper provides a unified approach to estimating the idealization error caused by defeaturing, dimension reduction or their combination. Simply using an approach either targeting defeaturing, or dimension reduction, or 


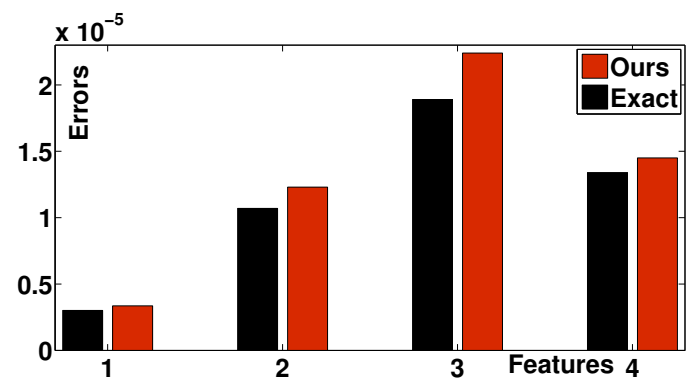

Figure 10: Idealization error estimation results for the problem in Figure 8.

simply summing the absolute values or the error due to each independently, are inadequate - we must also consider their interaction. Instead of an approximate error estimate, we build error bounds, which are important have confidence in the results. Our experimental results indicate that our error estimates both bound the true error and have an effectivity quite close to 1 .

On the other hand, the proposed approach to idealization error estimation assumes that the solution $u_{0}$ is exactly known. However, in practical engineering analysis, solution $u_{0}$ is only computed approximately as a numerical solution $u_{0}^{h}$. We actually should seek an estimate of the error

$$
e^{h}=Q(u)-Q\left(u_{0}^{h}\right) .
$$

Noticing that

$$
e^{h}=Q(u)-Q\left(u_{0}^{h}\right)=\left(Q(u)-Q\left(u_{0}\right)\right)+\left(Q\left(u_{0}\right)-Q\left(u_{0}^{h}\right)\right),
$$

we can thus compute $e^{h}$ as a summation between the computed idealization error $Q(u)-Q\left(u_{0}\right)$ and the approximation error $Q\left(u_{0}\right)-Q\left(u_{0}^{h}\right)$. Estimation of the latter error can be achieved using various well developed techniques, for example, as reviewed in [12]. 


\section{Acknowledgements}

This work described in this paper was partially supported by the National Basic Research Program of China (2011CB302400), the NSF of China (61103103).

\section{References}

[1] S. Lee, Feature-based multiresolution modeling of solids, ACM T GRAPHIC 24 (4) (2005) 1417-1441.

[2] A. Thakur, A. Banerjee, S. Gupta, A survey of CAD model simplification techniques for physics-based simulation applications, ComputAided Des 41 (2) (2009) 65-80.

[3] K. Lee, C. Armstrong, M. Price, J. Lamont, A small feature suppression/unsuppression system for preparing B-Rep models for analysis, in: Proceedings of the ACM Symposium on Solid and Physical Modeling, Cambridge, MA, 2005, pp. 113-124.

[4] R. Ferrandes, P. Marin, J. Leon, F. Giannini, A posteriori evaluation of simplification details for finite element model preparation, Comput Struct 87 (1-2) (2009) 73-80.

[5] D.-P. Sheen, T. geun Son, D.-K. Myung, C. Ryu, S. H. Lee, K. Lee, T. J. Yeo, Transformation of a thin-walled solid model into a surface model via solid deflation, Comput-Aided Des 42 (8) (2010) 720-730. 
[6] T. Robinson, C. Armstrong, R. Fairey, Automated mixed dimensional modelling from 2D and 3D CAD models, Finite Elem Anal Des 47 (2) (2011) 151-165.

[7] D. White, S. Saigal, S. Owen, Meshing complexity of single part CAD models, in: Proceedings of the 12th International Meshing Roundtable Conference, Santa Fe, New Mexico, U.S.A., 2003.

[8] Y. Saad, Iterative methods for sparse linear systems, Halstead Press, 2003.

[9] Y. Bazilevs, V. Calo, J. Cottrell, J. Evans, T. Hughes, S. Lipton, M. Scott, T. Sederberg, Isogeometric analysis using T-splines, Comput Methods Appl Mech Engng 199 (5-8) (2010) 229-263.

[10] G. Foucault, J. Cuilliere, V. Francois, J. Leon, R. Maranzana, Adaptation of CAD model topology for finite element analysis, Comput-Aided Des 40 (2) (2008) 176-196.

[11] L. Fine, L. Remondini, J. Leon, Automated generation of FEA models through idealization operators, Int J Numer Methods Engng 49 (1-2) (2000) 83-108.

[12] T. Gratsch, K. Bathe, A posteriori error estimation techniques in practical finite element analysis, Comput \& Struct 83 (4-5) (2005) 235-265.

[13] J. Oden, S. Prudhomme, Estimation of modeling error in computational mechanics, J Comput Phys 182 (2002) 496-515. 
[14] E. Stein, S. Ohnimus, Coupled model- and solution-adaptivity in the finite-element method, Comput Methods Appl Mech Eng 150 (1-4) (1997) 327-350.

[15] A. V. D. Krasnov, T. Boeck, B. Knaepen, Modeling and discretization errors in large eddy simulations of hydrodynamic and magnetohydrodynamic channel flows, J Comput Phys 230 (5) (2011) 1903-1922.

[16] T. Hughes, J. Cottrella, Y. Bazilevsa, Isogeometric analysis: CAD, finite elements, NURBS, exact geometry and mesh refinement, Comput Methods Appl Mech Engng 194 (39-41) (2005) 4135-4195.

[17] W. R. Quadros, S. J. Owen, Defeaturing CAD models using a geometrybased size field and facet-based reduction operators, in: Proceedings of the 18th International Meshing Roundtable, 2009, pp. 301-328.

[18] V. Shapiro, I. Tsukanov, A. Grishin, Geometric issues in computer aided design/computer aided engineering integration, J Comput Inf Sci Eng 11 (2) (2011) 021005-1:13.

[19] K. Choi, N. Kim, Structural Sensitivity Analysis and Optimization I: Linear Systems, Springer, New York, 2005.

[20] J. Sokolowski, A. Zochowski, On topological derivative in shape optimization, SIAM J Control Optim 37 (4) (1999) 1251-1272.

[21] S. Nazarov, Asymptotic analysis of shape functionals, Journal de Mathématiques Pures et Appliqués 82 (2) (2003) 125-196. 
[22] I. Turevsky, S. Gopalakrishnan, K. Suresh, Defeaturing: A posteriori error analysis via feature sensitivity, Int J Numer Methods Engng 76 (9) (2008) 1379-1401.

[23] S. Gopalakrishnan, K. Suresh, Feature sensitivity: A generalization of topological sensitivity, Finite Elem Anal Des 44 (11) (2008) 696-704.

[24] M. Ainsworth, M. Arnold, Computable error bounds for some simple dimensionally reduced models on thin domains, IMA J Numer Anal 21 (1) (2001) 81-105.

[25] K. Vemaganti, Local error estimation for dimensionally reduced models of elliptic boundary value problems, Comput Methods Appl Mech Engng $192(1-2)(2003) 1-14$.

[26] K. Vemaganti, N. Billade, Local error estimation for dimensional reduction: application to special geometries, Commun Numer Methods Eng 20 (4) (2004) 323-333.

[27] N. Billade, K. Vemaganti, Hierarchical models of thin elastic structures: Overview and recent advances in error estimation and adaptivity, Comput Methods Appl Mech Engng 196 (2007) 3508-3523.

[28] I. Turevsky, S. Gopalakrishnan, K. Suresh, An efficient numerical method for computing the topological sensitivity of arbitrary-shaped features in plate bending, Int J Numer Methods Engng 79 (13) (2009) $1683-1702$. 
[29] M. Li, S. Gao, R. Martin, Estimating effects of removing negative features on engineering analysis, Comput-Aided Des 43 (1) (2011) 14021212 .

[30] R. Becker, R. Rannacher, A feed-back approach to error control in finite element methods: basic analysis and examples, East-West J Numer Math 4 (1996) 237-264.

[31] W. Bangerth, R. Rannacher, Adaptive finite element methods for differential equations, Basel: Birkhauser, 2003.

[32] B. Smith, P. Bjorstad, W. Gropp, Domain Decomposition: Parallel Multilevel Methods for Elliptic Partial Differential Equations, Cambridge University, Cambridge, 2004.

[33] M. Giles, E. Suli, Adjoint methods for PDEs: a posteriori error analysis and postprocessing by duality, Acta Numer 11 (2002) 145-236.

[34] M. Giles, N. Pierce, An introduction to the adjoint approach to design, Flow, Turbul Combust 65 (2000) 393-415.

[35] P. DAVIS, Interpolation and Approximation, Blaisdell, 1963.

[36] J. Oden, K. Vemaganti, Estimation of local modeling error and goaloriented modeling of heterogeneous materials; Part I: error estimates and adaptive algorithms, J Comput Phys 164 (1) (2000) 22-47.

[37] COMSOL multiphysics 3.5a, http://www.comsol.com. (2007). 
[38] S. Prudhomme, J. Oden, T. Westermann, J. Bass, M. Botkin, Practical methods for a posteriori error estimation in engineering applications, Int J Numer Methods Engng 56 (2003) 1193-1224.

[39] M. Giles, E. Suli, An introduction to a posteriori error analysis and adjoints, Tech. rep., NASA Ames Research Center, CA, and The von Karman Institute for Fluid Dynamics, Belgium (2001).

[40] D. Estep, A posteriori error bounds and global error control for approximations of ordinary differential equations, SIAM J Numer Anal 32 (1995) 1-48.

[41] K. Bletzinger, M. Bischoff, E. Ramm, A unified approach for shearlocking-free triangular and rectangular shell finite elements, Comput \& Struct 75 (3) (2000) $321-334$. 